\title{
Los contratos de investigación y desarrollo en el texto refundido de la Ley de Contratos del Sector Público
}

\author{
Manuel Mesa Vila \\ Abogado experto en contratación pública \\ Profesor asociado Universidad Loyola Andalucía
}

\begin{abstract}
El contrato de investigación y desarrollo es un contrato del sector público típico recogido como tal en el anexo II del Real Decreto Legislativo 3/2011, de 14 de noviembre, por el que se aprueba el texto refundido de la Ley de Contratos del Sector Público, (TRLCSP, en lo sucesivo) como una categoría de contrato de servicios, y por tanto, incluido en su ámbito objetivo de aplicación. No obstante, esta afirmación que a priori parece simple está plagada de matices y excepciones a lo largo del articulado que hacen del contrato de I + D uno de los de más compleja regulación y, por tanto, proclive a generar confusión y consecuentemente grandes dosis de inseguridad jurídica.

En el presente artículo se analizan las diferentes figuras de contratos de I + D mencionados en el texto refundido, realizándose una primera delimitación elemental de contratos excluidos y contratos incluidos de la ley, para analizar a continuación los elementos diferenciadores de cada una de las categorías que resultará fundamental para establecer las normas y procedimiento a seguir en lo relativo a la publicidad de la licitación y a la forma de adjudicar el contrato.
\end{abstract}

\section{INTRODUGCIÓN}

Bajo el término genérico de contratos de investigación y desarrollo se han ido incluyendo una serie de negocios y relaciones jurídicas distintas, que en su conjunto podemos definir como el contrato en virtud del cual una empresa (o una universidad, spin-off, centro de investigación, instituto, etc.) se compromete a la ejecución de un proyecto de $\mathrm{I}+\mathrm{D}$ a petición en este caso de un organismo público. El proyecto se realiza en base a unos objetivos que se pretenden conseguir, con un plan de trabajo, unas condiciones económicas determinadas, 
un equipo de investigadores definido y un clausulado que puede variar mucho dependiendo de las características del proyecto. El contrato de I+D debe suponer la generación de un nuevo conocimiento o una innovación.

Podemos afirmar que, por regla general, los contratos de investigación son contratos de actividad (jurídicamente denominados de arrendamiento de servicios, artículo 1544 del Código Civil). En este tipo de contratos, la obligación principal del investigador no consiste en alcanzar un resultado sino en el desarrollo de una actividad, dado que la obtención del resultado depende de factores ajenos a la voluntad del que lo presta, con lo que la incertidumbre juega un papel fundamental que debe ser regulado en el contrato. El hecho de que se tengan que presentar cada cierto tiempo informes de las tareas que se van ejecutando, no significa que se prometa resultado alguno, su único objeto es comprobar el estado efectivo de los trabajos de investigación. En virtud del contrato, el investigador se compromete a asignar unos medios humanos y materiales para llevar a cabo una serie de tareas concretas. Esta consideración de contrato de medios y no de resultados, tiene gran trascendencia a la hora de determinar los derechos, obligaciones y responsabilidades de organismo contratante y de la empresa, instituto o universidad contratados.

Como hemos indicado en el resumen inicial, en principio el contrato de I $+\mathrm{D}$ es un contrato que aparece expresamente mencionado en el anexo II del TRLCSP como una categoría de contrato de servicios, y por tanto, incluido en su ámbito objetivo de aplicación. No obstante, esta afirmación que a priori parece simple está plagada de matices y excepciones a lo largo del articulado que hacen del contrato de I + D uno de los de más compleja regulación.

El creciente interés de las administraciones pública por la búsqueda de soluciones innovadoras para la provisión de necesidades públicas unido al notable incremento de entidades, sociedades, fundaciones, institutos, públicos o semipúblicos dedicados a la investigación y la innovación tecnológica, son factores que han contribuido a la proliferación de contratos públicos de I + D cuya regulación en el ámbito de una norma de carácter administrativo, cargada de formalismos y procedimientos reglados de carácter obligatorio, chocan en muchas ocasiones con la propia esencia de estos contratos basados en la incertidumbre y en la oportunidad. Motivo por el cual el legislador ha visto necesario matizar mucho las diferentes tipologías de contratos de I + D y modular el grado de aplicación del TRLCSP tomando como base el tratamiento de cada uno de los elementos del contrato: sujetos, objeto y causa.

Para determinar si nos encontramos ante un contrato incluido o excluido 
del ámbito objetivo del TRLCSP es necesario tener en cuente tres factores que resultan fundamentales: en primer lugar, la financiación o remuneración del contrato, cual de las dos partes lo paga, en segundo lugar, y muy especialmente el tratamiento y propiedad de los resultados que se obtengan; si estos se comparten entre las dos partes, si se reserva su titularidad la parte contratante para una posterior difusión o si se reserva el resultado para su utilización exclusiva en su actividad propia y por último cual de las dos partes asume el riesgo y ventura de la ejecución del contrato, es decir si hay pérdidas económicas en la ejecución del contrato quien asume esas pérdidas y si hay excedentes, a quien corresponden esos excedentes.

\section{CONTRATOS Y NEGOGIOS JURÍDICOS EXGLUIDOS DEL TRLCSP}

El artículo 4 establece una amplia enumeración de los contratos y negocios excluido del ámbito objetivo del TRLCSP. En relación a los contratos de I+D nos encontramos los siguientes:

\subsection{Convenios de I+D}

El artículo 4.1 del TRLCSP dispone:

Están excluidos del ámbito de la presente Ley los siguientes negocios y relaciones jurídicas:

d) Los convenios que, con arreglo a las normas específicas que los regulan, celebre la Administración con personas fisicas o jurídicas sujetas al derecho privado, siempre que su objeto no esté comprendido en el de los contratos regulados en esta Ley o en normas administrativas especiales.

La utilización de la figura del convenio de colaboración ha sido una práctica muy extendida para el desarrollo de proyectos de I+D. No obstante, un convenio es una relación jurídica que no tiene naturaleza contractual por la ausencia del carácter oneroso propia de los contratos, razón que justifica su exclusión del ámbito objetivo del texto refundido. En los convenios, tanto la finalidad que se pretende cubrir como la relación jurídica que se entabla entre las partes son completamente diferentes a la de los contratos. Muchas veces este importante matiz se olvida y erróneamente se pretende salvar con un simple cambio de denominación, llamando convenio a los que a todas luces es un contrato. 
La nota elemental del convenio es que las partes (no necesariamente dos, pueden ser más de dos) tienen un objetivo común, quieren hacer o conseguir algo que cada una de las partes por separado no es capaz de realizar por lo que se unen para poner en común los recursos de que disponen en pos de ese objetivo común. No se trata de una relación simple en la que una parte paga y la otra hace, en el convenio todos hacen o aportan y todos se benefician, siendo fundamental en este marco de relaciones el equilibrio: lo que cada parte aporta debe ser más o menos homogéneo.

En el caso concreto de los convenios de I $+\mathrm{D}$, todas las partes tienen interés en desarrollar un proyecto, para lo cual cada uno aportará los medios de que pueda disponer; medios humanos, materiales, instalaciones, know how, y por supuesto, dinero. En los convenios no se debe poder distinguir una relación sinalagmática, en la que existan dos obligaciones básicas una de pagar y una de hacer.

Por supuesto, constituye una nota esencial de los convenios de colaboración el hecho de que los resultados han de beneficiar a todas las partes y su titularidad debe ser compartida por todos los firmantes.

Además de todas estas exigencias referidas al tipo de relación que se establece entre las partes, existe otra limitación que resulta determinante cual es que su objeto no esté comprendido en el de los contratos regulados en esta Ley o en normas administrativas especiales. Se trata de una limitación dificil de salvar habida cuenta que el anexo II del TRLCSP, recoge expresamente en la categoría 8 a los contratos de investigación y desarrollo, como un tipo de contratos de servicio regulados en la ley.

Es la nota marginal del anexo II, referida a la categoría 8 la que de una forma muy confusa y con una redacción que bien parece un acertijo, excluye los convenios de colaboración y los diferencia de la figura de los contratos de I+D:

Exceptuando los servicios de investigación y desarrollo distintos de aquellos cuyos resultados corresponden al poder adjudicador y/o a la entidad adjudicadora para su uso exclusivo, siempre que este remunere integramente la prestación del servicio.

Se trata de una excepción importada directamente de la Directiva 18/2004/CE, artículo 16.f, que viene a reiterar dos de los elementos esenciales que diferencian a los convenios de los contratos en los que la propiedad de los resultados y la remuneración del servicio corresponden en exclusiva al poder adjudicador comprador. 
Resulta muy ilustrativa la Recomendación 8/2008, de 13 de mayo, Comisión Consultiva de Contratación Pública de Andalucía, sobre los convenios de colaboración con las entidades públicas y personas físicas o jurídicas sujetas al derecho privado, en especial sobre los convenios de desarrollo e investigación y su distinción de los contratos de servicios, que en sus conclusiones afirma lo siguiente:

Sólo resultaría procedente la suscripción de convenios de colaboración en materia de $I+D$ en los casos en que concurran las siguientes notas:

1.- Todas las partes que lo suscriben tienen un interés común en llevar a cabo un proyecto conjunto. La existencia de este interés común se aprecia cuando, con los resultados científicos que se deriven de dicho proyecto, se da satisfacción a las necesidades de cada una de las partes, de acuerdo con sus objetivos y fines. No puede considerarse que existe ese interés común cuando el interés de una de las partes consista en la realización del trabajo y que éste le sea sufragado -en todo o en parte-por enmarcarse ello en la actividad propia de la entidad

2.- El objeto del convenio no se traduce en prestaciones y contraprestaciones de las partes y no consiste en la financiación de un proyecto sino en la realización del mismo, de tal forma que, todas las partes contribuyen al desarrollo del proyecto poniendo en común los datos, conocimientos y elementos personales y materiales con que cuenten.

3.- El proyecto debe generar un resultado cientifico del que se beneficien todas las partes colaboradoras y del que hagan o puedan hacer uso todas ellas.

4.- que su objeto no esté comprendido en el de los contratos regulados en la Ley o en normas administrativas especiales.

\subsection{Los contratos de I + D celebrados por los OPI}

Los contratos de servicios y suministro celebrados por los Organismos Públicos de Investigación estatales y los Organismos similares de las Comunidades Autónomas que tengan por objeto prestaciones o productos necesarios para la ejecución de proyectos de investigación, desarrollo e innovación tecnológica o servicios técnicos, cuando la presentación y obtención de resultados derivados de los mismos esté ligada a retornos científicos, tecnológicos o industriales susceptibles de incorporarse al tráfico jurídico y su realización haya sido encomendada a equipos de investigación del Organismo mediante procesos de concurrencia competitiva. (Artículo 4.1.q)

Aquí se trata de la contratación de suministros y servicios necesarios para la 
ejecución de proyectos de investigación y desarrollo en el que podría perfectamente encajar un contrato de I+D parcial de ese proyecto. Se trata de una exclusión del ámbito del TRLGSP tremendamente restrictiva tanto en el aspecto subjetivo como en los elementos objetivos que han de concurrir.

En lo que al aspecto subjetivo se refiere, sólo es aplicable a los OPI estatales y a los organismos similares de las Comunidades Autónomas. Los OPI estatales son sólo y exclusivamente los definidos como tales y enumerados en la Ley de la Ciencia de 1986, que otorgó el estatuto de OPI a los seis grandes centros de investigación adscritos a diversos ministerios: el Consejo Superior de Investigaciones Científicas, el Centro de Investigaciones Energéticas, Medioambientales y Tecnológicas, el Instituto Geológico y Minero de España, el Instituto Nacional de Tecnología Aeroespacial, el Instituto Español de Oceanografía y el Instituto Nacional de Investigaciones Agrarias. Posteriormente se unió a estos centros el Instituto de Salud Carlos III.

En el ámbito del Estado, la delimitación subjetiva de este supuesto está muy clara. Pero, como hemos visto, el artículo lo hace extensible a los organismos similares de las comunidades autónomas. Por lo que habrá que estar a lo que disponga cada comunidad y lo que cada una entienda que es un OPI.

A título de ejemplo podemos citar que la Comunidad Autónoma de Aragón, exige para considerar a un determinado organismo como OPI autonómico, que se cree por Ley del Parlamento autonómico, en aplicación de lo dispuesto en el artículo 66 del Decreto Legislativo 2/2001, de 3 de julio, del Gobierno de Aragón, por el que se aprueba el Texto Refundido de la Ley de la Administración de la Comunidad Autónoma de Aragón (En este sentido, Informe 4/2008, de 15 de mayo, de la Junta consultiva de Contratación Administrativa de Aragón).

En Andalucía, solo hace extensible la calificación de OPI exclusivamente al Instituto Andaluz de Investigación y Formación Agraria, Pesquera, Alimentaria y de la Producción Ecológica (IFAPA), creado por la Ley 1/2003 (artículo 32 de la Ley 16/2007, de 3 de diciembre, Andaluza de la Ciencia y el Conocimiento).

Por último, añadir que la Disposición Final 3a , Ocho de la Ley 14/2011 de la Ciencia incorporó a este respecto una importante novedad que amplía el espectro de organismos que pueden aplicar esta importante excepción del artículo 4.1.q e incluye en el concepto a las universidades públicas: 
A los efectos previstos en el párrafo q) del artículo 4 de la Ley 30/2007, de 30 de octubre, de Contratos del Sector público, las Universidades tendrán la consideración de Organismo Publico de Investigación

La suma de requisitos acumulativos que tienen que concurrir en el contrato en cuestión se puede ver de forma más gráfica en el siguiente cuadro:

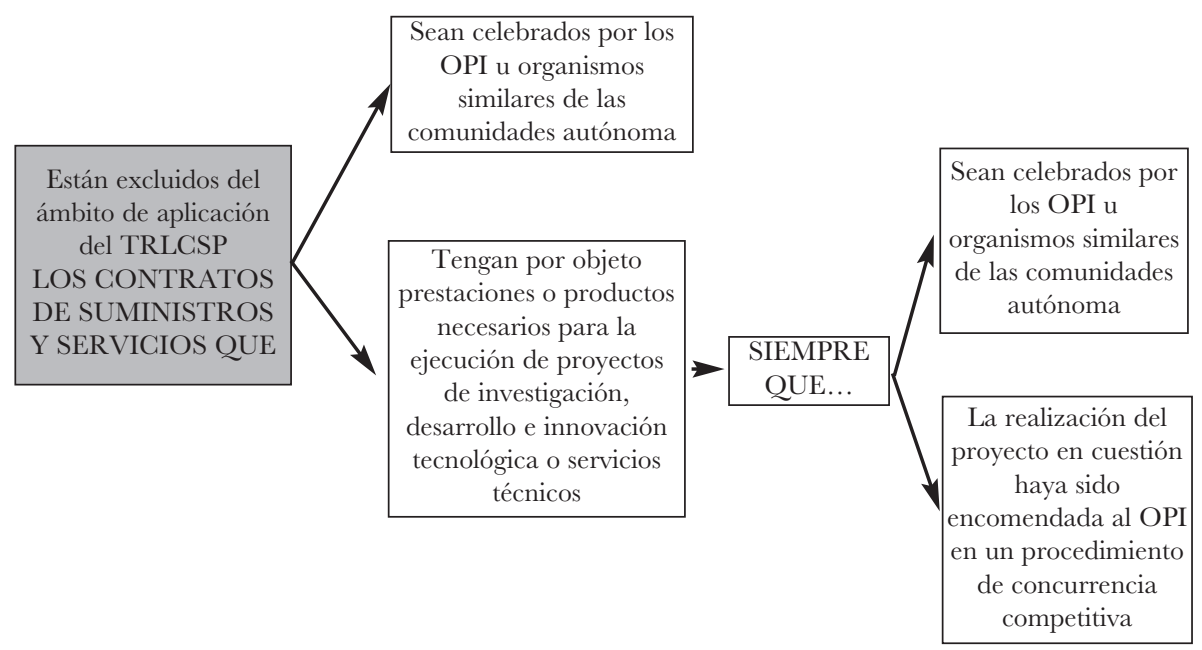

\subsection{Los contratos de I + D (compra pública precomercial)}

Los contratos de investigación y desarrollo remunerados íntegramente por el órgano de contratación, siempre que éste comparta con las empresas adjudicatarias los riesgos y los beneficios de la investigación científica y técnica necesaria para desarrollar soluciones innovadoras que superen las disponibles en el mercado. En la adjudicación de estos contratos deberá asegurarse el respeto a los principios de publicidad, concurrencia, transparencia, confidencialidad, igualdad y no discriminación y de elección de la oferta económicamente más ventajosa. (Artículo 4.1.r)

Se trata de la exclusión de los contratos llamados a cubrir una de las vertientes de la compra pública innovadora: la denominada compra pública precomercial. Los contratos del 4.1.r deben tener por objeto la investigación y desarrollo de soluciones innovadoras que superen las disponibles en el mercado. 
Esta excepción fue introducida en la LCSP por la Ley 2/2011, de Economía Sostenible, que además para incentivar el uso de esta nueva modalidad de contratos, establecía el compromiso del Consejo de Ministros de fijar dentro de los presupuestos de cada departamento ministerial y de cada organismo público vinculado con o dependiente de la Administración General del Estado, las cuantías destinadas a la financiación de contratos a los que hace referencia este.

En este caso el contrato es íntegramente remunerado por el órgano de contratación, pero el elemento diferencial esencial que provoca que estos contratos queden excluidos la ley es que contratante y contratado comparten el riego y ventura del contrato. Diferenciándose así del resto de contratos del sector público en los que el riesgo y ventura del empresario constituye uno de sus elementos más característicos (artículo 215 TRLCSP).

A pesar de la exclusión de su ámbito de aplicación, la ley somete la adjudicación de estos contratos a los principios de publicidad, concurrencia, transparencia, confidencialidad, igualdad y no discriminación y de elección de la oferta económicamente más ventajosa. Si bien la forma concreta de aplicación de estos principios quedará al criterio y forma que establezca el órgano de contratación. Tratándose de poderes adjudicadores que no tengan el carácter de administración pública vendrán obligados a aplicar sus correspondientes instrucciones internas de contratación (Artículo 191) dado que es el documento que establece la forma en que esa entidad concreta garantiza la aplicación de lo mencionados principios rectores de la contratación pública.

\section{CONTRATOS Y NEGOGIOS JURÍDICOS INGLUIDOS}

Se encuentran incluidos en el ámbito objetivo de aplicación los contratos de servicio de investigación y desarrollo (categoría 8 del anexo II) que no reúnan las características y elementos que hemos visto que justifican su exclusión.

Los elementos esenciales del contrato de I + D incluidos en el TRLCSP son los siguientes:

- Son remunerados o pagados íntegramente por el poder adjudicador.

- Se ejecutan a riesgo y ventura del empresario.

Los resultados obtenidos son propiedad del poder adjudicador comprador. 
El régimen jurídico de los contratos de $\mathrm{I}+\mathrm{D}$ es el régimen general de los contratos de servicio, si bien existe una importante matización en los contratos de I + D sujetos a regulación armonizada, ya que el artículo 13.1 del TRLCSP considera contratos SARA a los contratos de servicios comprendidos en las categorías 1 a 16 del Anexo II, cuyo valor estimado sea igual o superior a 200.000 $€$. No obstante el artículo 13.2 determina una importante excepción:

No obstante lo señalado en el apartado anterior, no se consideran sujetos a regulación armonizada, cualquiera que sea su valor estimado, los contratos siguientes:

b) Los de investigación y desarrollo remunerados integramente por el órgano de contratación, siempre que sus resultados no se reserven para su utilización exclusiva por éste en el ejercicio de su actividad propia.

La distinción se hace descansar en este caso en otro concepto con un alto grado de indefinición cual es el tratamiento que se de a los resultados. Se parte de la base de que el órgano de contratación es el propietario único de los resultados derivados de los servicios de investigación y desarrollo que se han contratado, pero el matiz se produce a la hora de determinar el tratamiento o el destino que se dará a esos resultados: si son para el uso exclusivo del órgano contratante en el ejercicio de su actividad propia (sumado a que el valor estimado del contrato es superior a los 130.000 ó $200.000 €$, en función de cual sea el órgano convocante) será un contrato sujeto a regulación armonizada, si esta premisa no se cumple, es decir, si el resultado no se reserva para su utilización exclusiva será un contrato ordinarios no SARA.

Se trata de un distinción fundamental desde el punto de vista del procedimiento a seguir para la adjudicación del contrato (sobre todo en cuanto a su publicidad y plazos) que descansa sobre una premisa muy débil y que en la mayoría de los casos dado el carácter de los órganos de contratación y el inherente interés público que preside su actuación, nos encontraremos la excepción del artículo 13.2.b), no siendo, por tanto, contratos sujetos a regulación armonizada, dado que lo más normal será que el órgano de contratación no se reserve los resultados para su utilización exclusiva sino que los destine a la mejora del conocimiento y de la ciencia del aspecto concreto que se investigue, dotándolo de valor público añadido y revertiendo el resultado obtenido a la sociedad en su conjunto.

Los pliegos reguladores de la licitación deben, por tanto regular de forma clara el tratamiento y el destino que se dará a los resultados obtenidos, siendo jurídicamente exigible que una vez concluido el contrato el resultado se difunda y se comparta de forma efectiva. 
Se adjunta a este artículo un cuadro sinóptico que incluye los elementos principales para la distinción de las distintas modalidades de contrato de I + D estudiadas en este artículo.

\section{CUADRO RESUMEN DEL TRATAMIENTO DE LOS GONTRATROS DE I + D EN EL TRLGSP}

\begin{tabular}{|c|c|c|c|c|c|c|}
\hline & & $\begin{array}{l}\text { REMUNERACIÓN } \\
\text { FINANCIACION } \\
\text { DEL CONTRATO }\end{array}$ & RESULTADOS & $\begin{array}{l}\text { RIESGO Y } \\
\text { VENTURA } \\
\text { DEL CON- } \\
\text { TRATO }\end{array}$ & $\begin{array}{l}\text { REGULA- } \\
\text { CIÓN }\end{array}$ & $\begin{array}{c}\text { OTRAS } \\
\text { CARACTERISTI- } \\
\text { CAS ESPECIALES }\end{array}$ \\
\hline \multirow{3}{*}{$\begin{array}{l}\text { CONTRATOS } \\
\text { Y NEGOCIOS } \\
\text { JURÍDICOS } \\
\text { EXLUIDOS } \\
\text { DEL TRLCSP }\end{array}$} & $\begin{array}{c}\text { CONVENIOS } \\
\text { I + D }\end{array}$ & $\begin{array}{l}\text { Repartida entre los } \\
\text { firmantes del conve- } \\
\text { nio. Puede ser que se } \\
\text { aporten medios y no } \\
\text { dinero. }\end{array}$ & $\begin{array}{c}\text { Compartidos en to- } \\
\text { do caso }\end{array}$ & Compartido & $\begin{array}{l}\text { Art. 4.1.d } \\
\text { TRLCSP y } \\
\text { Anexo II }\end{array}$ & $\begin{array}{l}\text { - No tienen naturale- } \\
\text { za contractual } \\
\text { - Pueden ser más de } \\
\text { dos partes }\end{array}$ \\
\hline & $\begin{array}{c}\text { CONTRATOS } \\
\text { I + D } \\
\text { OPI'S }\end{array}$ & $\begin{array}{l}\text { Proyectos encomen- } \\
\text { dados en procesos de } \\
\text { concurrencia compe- } \\
\text { titiva }\end{array}$ & $\begin{array}{l}\text { Resultados ligados a } \\
\text { retornos científicos o } \\
\text { tecnológicos suscep- } \\
\text { tibles de incorporar- } \\
\text { se al tráfico jurídico }\end{array}$ & No se especifica & $\begin{array}{l}\text { Art. 4.1.q } \\
\text { TRLCSP }\end{array}$ & $\begin{array}{l}\text { El ámbito de aplica- } \\
\text { ción de esta excep- } \\
\text { ción se restringe en } \\
\text { exclusiva a los OPIS } \\
\text { y organismos simila- } \\
\text { res de las CCAA }\end{array}$ \\
\hline & $\begin{array}{c}\text { CONTRATOS } \\
\text { I + D } \\
\text { COMPRA PÚ- } \\
\text { BLICA PRE- } \\
\text { COMERCIAL }\end{array}$ & $\begin{array}{c}\text { Íntegramente por el } \\
\text { órgano de contrata- } \\
\text { ción }\end{array}$ & $\begin{array}{c}\text { Compartidos. } \\
\text { Deben consistir en } \\
\text { soluciones innovado- } \\
\text { ras }\end{array}$ & Compartido & $\begin{array}{l}\text { Art. 4.1.r } \\
\text { TRLCS }\end{array}$ & $\begin{array}{l}\text { Sometidos a los prin- } \\
\text { cipios de publicidad, } \\
\text { concurrencia, trans- } \\
\text { parencia, igualdad y } \\
\text { elección de la oferta } \\
\text { económicamente } \\
\text { más ventajosa }\end{array}$ \\
\hline \multirow{2}{*}{$\begin{array}{c}\text { CONTRATOS } \\
\text { Y NEGOCIOS } \\
\text { JURÍDICOS } \\
\text { INCLUIDOS } \\
\text { EN EL } \\
\text { TRLCSP }\end{array}$} & $\begin{array}{c}\text { CONTRATOS } \\
\text { I + D } \\
\text { NO SARA }\end{array}$ & $\begin{array}{c}\text { Íntegramente por el } \\
\text { órgano de contrata- } \\
\text { ción }\end{array}$ & $\begin{array}{c}\text { No se reservan para } \\
\text { la utilización exclusi- } \\
\text { va del órgano con- } \\
\text { tratante en el ejerci- } \\
\text { cio de su actividad } \\
\text { propia }\end{array}$ & $\begin{array}{c}\text { Del empresario } \\
\text { régimen general } \\
\text { Art. 215) }\end{array}$ & $\begin{array}{c}\text { Regulación ge- } \\
\text { neral Contrato } \\
\text { de servicio del } \\
\text { TRLCSP y } \\
\text { Art. 13.2.b }\end{array}$ & \\
\hline & $\begin{array}{c}\text { CONTRATOS } \\
\text { I + D } \\
\text { SARA }\end{array}$ & $\begin{array}{c}\text { Íntegramente por el } \\
\text { órgano de contrata- } \\
\text { ción }\end{array}$ & $\begin{array}{c}\text { Se reservan para la } \\
\text { utilización exclusiva } \\
\text { del órgano contra- } \\
\text { tante en el ejercicio } \\
\text { de su actividad pro- } \\
\text { pia }\end{array}$ & $\begin{array}{c}\text { Del empresario } \\
\text { régimen general } \\
\text { Art. 215) }\end{array}$ & $\begin{array}{l}\text { Regulación ge- } \\
\text { neral Contrato } \\
\text { de servicio del } \\
\text { TRLCSP }\end{array}$ & \\
\hline
\end{tabular}

